\title{
Technè
}

La science au service de l'histoire de l'art et de la préservation des biens culturels

$44 \mid 2016$

Archives de l'humanité : les restes humains patrimonialisés

\section{Revisiter les restes humains grâce à la paléogénétique}

Revisiting human remains thanks to paleogenetics

\section{Céline Bon}

\section{(2) OpenEdition}

12 Journals

\section{Édition électronique}

URL : http://journals.openedition.org/techne/1054

DOI : 10.4000/techne.1054

ISSN : 2534-5168

\section{Éditeur}

C2RMF

\section{Édition imprimée}

Date de publication : 1 novembre 2016

Pagination : 64-66

ISBN : 978-2-7118-6339-6

ISSN : 1254-7867

Référence électronique

Céline Bon, «Revisiter les restes humains grâce à la paléogénétique », Technè [En ligne], 44 | 2016, mis en ligne le 19 décembre 2019, consulté le 26 juillet 2020. URL : http://journals.openedition.org/techne/ 1054 ; DOI : https://doi.org/10.4000/techne.1054

\section{(c) (i) (9)}

La revue Technè. La science au service de l'histoire de l'art et de la préservation des biens culturels est mise à disposition selon les termes de la Licence Creative Commons Attribution - Pas d'Utilisation

Commerciale - Pas de Modification 4.0 International. 


\section{Céline Bon}

\section{Revisiter les restes humains grâce à la paléogénétique}

Revisiting human remains thanks to paleogenetics

Résumé. En donnant accès au matériel génétique des échantillons archéologiques, la paléogénétique, ou étude de l’ADN ancien, a révolutionné la paléontologie et l'archéologie. Ces études, menées sur une molécule endommagée, nécessitent des précautions particulières afin de déterminer avec précision la séquence d'ADN portée par les individus anciens. Ainsi, le matériel génétique d'espèces disparues, comme celle de l'Homme de Néandertal, permet de retracer l'histoire évolutive de ces taxons et leurs relations avec l'Homme moderne. Les études paléogénétiques ont également montré que les migrations et les métissages entre populations humaines anciennes sont à l'origine de la diversité génétique actuelle.

Mots-clés. ADN, génétique, génome, archéologie, Néandertal, Homo sapiens.

\author{
Abstract. By giving access to the genetic material of archaeological \\ samples, paleogenetics, or the study of ancient DNA, has \\ revolutionized paleontology and archaeology. These studies, \\ conducted on a damaged molecule, required particular precautions \\ to be taken so as to determine the exact DNA sequence present \\ in ancient individuals. Thus, the genetic material of extinct \\ species, like that of Neanderthal Man, enables us to retrace the \\ evolution of these taxa and their relationship with Modern Man. \\ Paleogenetic studies have also shown that migrations \\ and the interbreeding of human populations are the reason \\ for present genetic diversity. \\ Keywords. DNA, genetics, genome, archaeology, Neanderthal, \\ Homo sapiens.
}

Depuis que l'on a découvert, durant les années 1980, que l'ADN pouvait se conserver durant des centaines, voire des milliers d'années, la paléogénétique, ou analyse de l'ADN ancien, s'est révélée un outil indispensable dans l'étude des restes animaux. L'ADN, cette molécule qui contient le patrimoine génétique d'un individu, est hérité de ses parents et accumule les mutations au cours du temps. Il permet donc de retracer l'histoire de nos ancêtres, soit récente (comme les relations de parenté au sein d'un groupe), soit plus ancienne (les relations phylogénétiques entre espèces). L'étude de l'ADN ancien donne accès à des informations génétiques concernant des espèces ou des populations avant qu'elles ne soient touchées par des événements marquants comme l'extinction ou le métissage avec d'autres populations. De plus, l'information codant l'apparence physique d'un individu se trouve dans le génome : c'est grâce à l'interaction entre environnement et matériel génétique qu'est défini le phénotype d'un individu.

\section{De l'échantillon archéologique à la séquence d'ADN}

Pour avoir accès à l'ADN, il est nécessaire de l'extraire d'un matériel d'origine organique. Les restes animaux les plus fréquemment retrouvés étant les dents et les os, ce sont souvent ces derniers qui sont analysés ; d'autres restes provenant de collections, comme les cheveux ou les poils, peuvent être sélectionnés, voire même des excréments fossiles (coprolithes). Un fragment de quelques centaines de milligrammes est alors réduit en poudre, puis l'ADN en est extrait chimiquement. Deux voies d'analyses peuvent ensuite être suivies. Dans le cas d'une analyse ciblée, un fragment du génome considéré comme d'intérêt est sélectionné et amplifié. C'est le produit de cette amplification qui est ensuite séquencé et analysé. Cette méthode est particulièrement adaptée afin de cibler un marqueur particulier du génome, soit pour déterminer la lignée à laquelle un individu appartient, soit pour étudier des mutations associées à un phénotype précis. L'autre approche consiste à séquencer la totalité de l'ADN présent dans un extrait d'ADN, y compris les molécules provenant de bactéries, de champignons ou de virus du sol. Cette méthode, plus coûteuse, est la seule qui permet d'avoir accès à la totalité du génome d'un individu, et donc, qui permet une meilleure précision dans l'étude de ses origines et de son génotype. Elle nécessite cependant une analyse bioinformatique poussée après le séquençage afin de pouvoir différencier l'information génétique endogène du reste des séquences. 


\section{Les difficultés de l'étude de l'ADN ancien}

\section{Les molécules d'ADN sont endommagées}

S'il est possible de retrouver de l'ADN dans des restes archéologiques, l'ADN est une molécule fragile : sa durée de conservation est limitée et va dépendre de nombreux facteurs externes (comme la température ou l'activité microbienne). Ainsi, dans des conditions extrêmement froides, il est possible de retrouver de l'ADN âgé de près d'un million d'années. En revanche, dans un climat tempéré, il ne subsiste dans le meilleur des cas que quelques dizaines de milliers d'années. De plus, il est alors fragmenté : reconstituer le génome (ou même des fragments de génome) revient à reconstruire un puzzle de centaines de millions de pièces. Des endommagements de l'ADN, qui constituent une signature de l'ADN ancien, modifient la séquence du génome.

\section{Éviter les contaminations par de l'ADN moderne}

L'étude de l'ADN ancien nécessite donc des précautions très particulières. L'ADN ancien est présent en faibles quantités et se trouve donc en compétition avec les molécules d'ADN moderne présentes dans l'air ambiant. Ce problème est particulièrement crucial quand l'échantillon est humain, de la même espèce que le manipulateur qui l'étudie. De nombreuses précautions permettent d'éviter les contaminations par l'ADN moderne : elles commencent sur le terrain, lors de la fouille, en prélevant le fragment osseux dans des conditions aseptiques, et elles se poursuivent au laboratoire, dans une pièce isolée des autres laboratoires manipulant de l'ADN moderne, dotée de surpression afin d'éviter toute entrée d'air potentiellement contaminé, décontaminée régulièrement (fig. 1). Cependant, il n'est pas toujours possible de suivre ces recommandations, en particulier quand les fragments proviennent de collections : dans ce cas, les ossements ont été

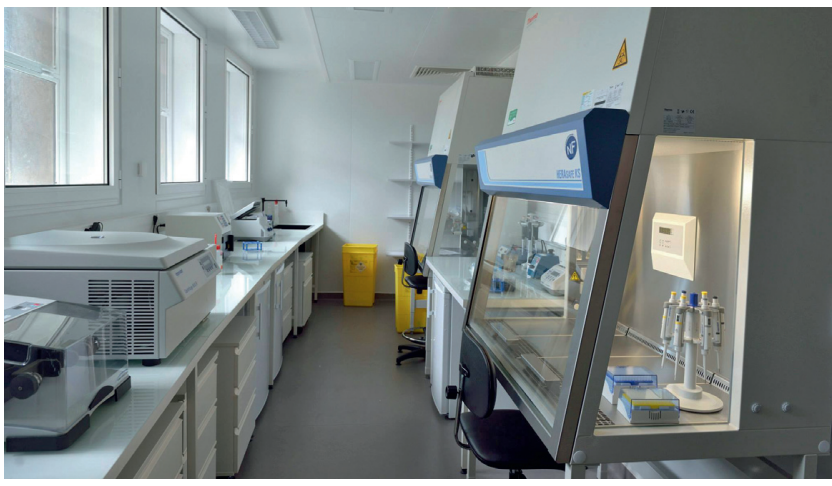

Fig. 1. Salle blanche dédiée à l'étude de l'ADN ancien du musée de l'Homme - Plateforme paléogénomique et génétique moléculaire humaine du Muséum national d'histoire naturelle. Une surpression à $+40 \mathrm{~Pa}$; un système de sas ; une exposition quotidienne aux UV ; des règles de comportement spécifiques permettent de limiter les contaminations par de l'ADN moderne. massivement manipulés avant l'analyse paléogénétique et il se révèle impossible de retracer toutes les personnes ayant pu laisser leurs traces ADN sur l'échantillon.

Les fragments d'ADN ancien sont alors identifiés a posteriori, en se fondant sur les profils d'endommagement typiques. C'est ce protocole qui a été utilisé pour étudier le génome d'un des plus anciens Hommes modernes hors d'Afrique, venant de la Grotte de Pestera cu Oase, ce qui a permis de montrer que cet individu était issu d'un métissage récent, vieux de seulement quelques générations, avec l'Homme de Néandertal.

D'autres méthodes permettent également de quantifier les contaminations et donc leur impact sur le résultat observé. Ainsi, si l'échantillon étudié provient d'une femme, aucun ADN correspondant au chromosome Y ne devrait y être trouvé. En revanche, si le spécimen est un homme, ne possédant donc qu'un seul chromosome X, toute hétérogénéité de séquence touchant cette portion du génome signale la présence d'une contamination.

Ainsi, même si la prévention des contaminations a priori est fondamentale dans l'étude de l'ADN ancien, des mesures a posteriori permettent de vérifier l'étendue des contaminations et de restreindre l'analyse aux fragments d'ADN endommagés et donc anciens.

Outre la contamination par de l'ADN humain moderne, les traitements appliqués aux échantillons entre leur découverte et l'arrivée au laboratoire d'ADN ancien peuvent être nocifs à la préservation de l'ADN. Ainsi, l'ADN étant une molécule soluble dans l'eau, le lavage des os tel qu'il est parfois effectué sur les chantiers de fouille peut diminuer de manière substantielle la quantité d'ADN ancien dans l'échantillon. D'autres traitements pratiqués couramment dans le passé, comme le vernissage des pièces osseuses, ont pu participer à la dégradation de l'ADN ancien. Même si des efforts sont désormais courants pour éviter tout traitement participant à la dégradation de l'ADN dans les collections d'anthropologie, il est encore difficile d'anticiper l'impact des traitements actuels, en particulier l'exposition aux rayons X, sur la préservation du matériel génétique.

\section{Des origines de l'Homme aux histoires des populations, apports de l'ADN ancien}

L'apport de l'ADN ancien à l'étude de l'évolution, et en particulier à l'histoire de l'Homme, est précieux. Parce qu'il donne accès au patrimoine génétique d'espèces ou de populations aujourd'hui éteintes, il a permis de resituer ces taxons dans la phylogénie des espèces animales, et de comparer les données obtenues à celles analysées depuis longtemps par la paléontologie. Ainsi, les positions phylogénétiques des Mammifères de l'ours des cavernes, du mégacéros ou du mammouth ont pu être déterminées grâce à des études paléogénétiques. De plus, en donnant accès à la diversité génétique diachronique d'espèces en train de s'éteindre, elle permet d'interroger le rôle des changements climatiques du Pléistocène ou de l'activité humaine sur la disparition des espèces. 


\section{Néandertal et Denisova}

Cependant, la plus grande percée effectuée grâce à l'étude de l'ADN ancien concerne notre compréhension de l'évolution de l'Homme. Entre la première extraction d'ADN ancien sur le spécimen type de l'Homme de Néandertal en 1997 et le séquençage du génome d'un Homo heidelbergensis de la Sima de los Uesos, l'histoire récente de la lignée humaine a été revisitée. L'étude de l'ADN ancien issu de restes humains indéterminés d'une grotte de l'Altai a permis d'enrichir la diversité taxonomique de la lignée humaine grâce à la découverte de l'Homme de Denisova. Mais surtout, la comparaison des génomes d'Hommes modernes et archaïques a mis en évidence une histoire complexe de métissages entre lignées humaines. Ainsi, l'évolution buissonnante de la lignée humaine se révèle être en fait un réseau complexe d'échanges génétiques.

\section{Les migrations à l'origine de la diversité génétique actuelle}

L'histoire de l'Homme moderne se trouve elle aussi enrichie par l'étude de l'ADN ancien (fig. 2). La paléogénétique, en donnant accès à la diversité génétique passée des populations humaines, permet de retracer les mouvements de populations ayant conduit aux populations actuelles. Pour l'instant, les

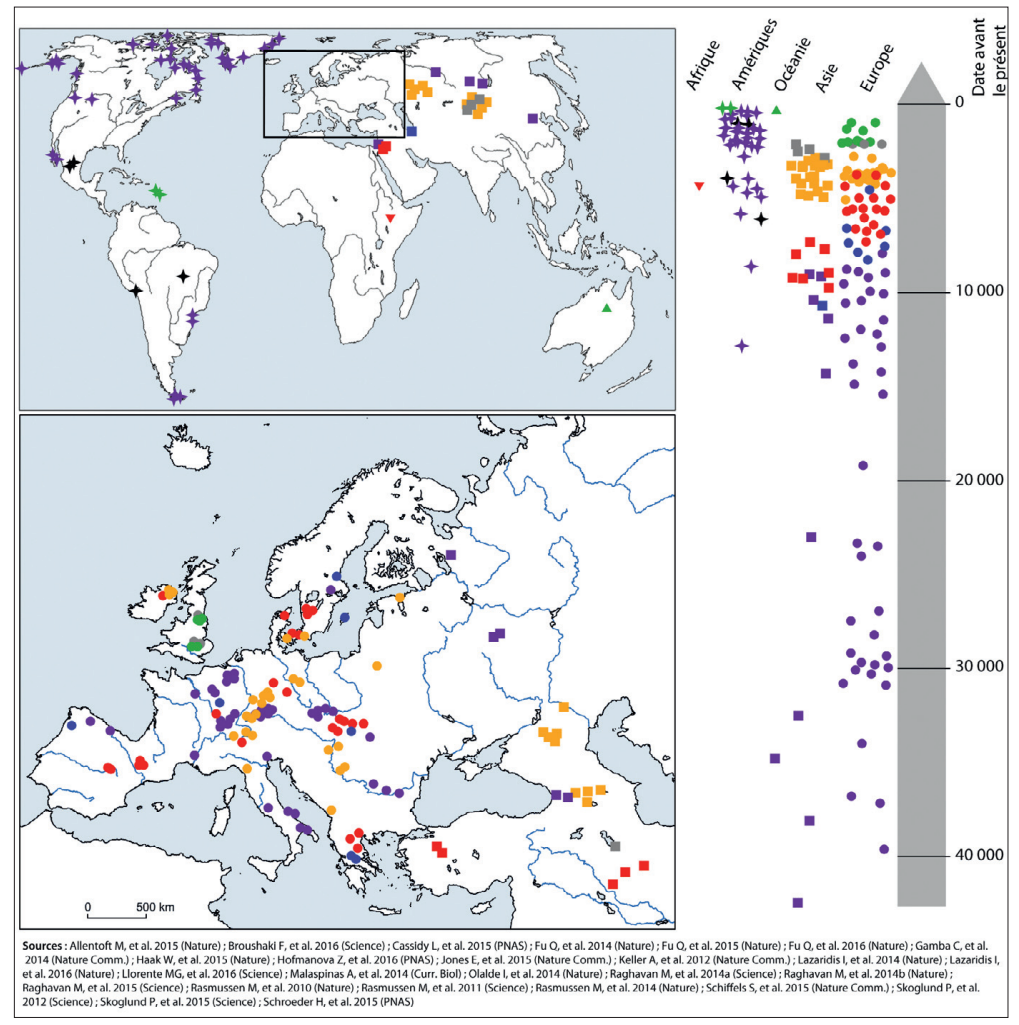

Fig. 2. Ensemble des échantillons humains pour lesquels des données génomiques sont disponibles. Les échantillons peuvent provenir d'un contexte paléolithique (violet), mésolithique (bleu), néolithique (rouge), de l'Âge du Bronze (orange), du Fer (gris) ou historique (vert). Ils proviennent majoritairement d'Europe (rond) et des Amériques (étoile), voire d'Asie (carré) dans lesquels l'ADN se conserve mieux. études se sont principalement concentrées sur le peuplement de l'Europe et de l'Amérique précolombienne. Dans ce dernier cas, la disparition de nombreuses populations amérindiennes et le métissage fréquent avec des populations originaires d'Europe ou d'Afrique rendent indispensables l'étude du matériel génétique de populations anciennes, souvent conservées dans des collections. Quant à l'Europe, des études diachroniques du patrimoine génétique de restes datant du Paléolithique à l'Âge du Fer ont permis de montrer que trois populations sont à l'origine des européens actuels : des chasseurs-cueilleurs du Mésolithique, des agriculteurs venus d'Anatolie au début du Néolithique et, à la fin du Néolithique, une population apparentée aux populations des steppes du nord de la mer Noire.

Ainsi, il est non seulement possible de retracer les migrations dans l'histoire de l'humanité, mais également de les dater précisément et d'associer les mouvements de populations avec les évolutions culturelles étudiées par les archéologues.

\section{L'ADN ancien et l'étude des sites archéologiques}

Par ailleurs, l'ADN, porteur de l'information génétique, permet de reconstituer les relations familiales ou l'apparence phénotypique. Ces informations nécessitent d'avoir accès au génome nucléaire : elles sont coûteuses à obtenir et donc rarement utilisées sur un grand nombre d'individus. Cependant, elles ont permis de montrer que les chasseurs-cueilleurs de la fin du Paléolithique supérieur ne possédaient pas les mutations associées aujourd'hui en Europe à une couleur de peau claire ou de retrouver la place du squelette attribué au roi anglais Richard III dans la généalogie de la famille royale d'Angleterre.

Ces découvertes ont principalement été possibles grâce à l'apport des nouvelles technologies de séquençages depuis une dizaine d'années. Ces technologies permettent d'avoir accès à l'ensemble du génome à un coût raisonnable, et non plus à de petites portions de gènes. Le génome, hérité de nos parents, donne non seulement des informations sur nos origines, sur notre apparence, mais également sur les structures sociales et les règles de mariage de nos ancêtres. Cette approche nécessite un génome complet de très bonne qualité, et donc un investissement financier important, et elle n'a pour l'instant été appliquée qu'à un faible nombre d'individus. Cependant, de nouveaux progrès technologiques laissent espérer qu'il sera bientôt possible d'appliquer des études paléogénétiques à des jeux de données comprenant davantage d'individus, y compris avec de l’ADN plus dégradé. 